\title{
Validación de la Escala de Emociones hacia el Cuidado del Agua
}

\author{
Validation of the Scale of Emotions toward Water Conservation Behavior
}

\author{
Juan Carlos Manríquez-Betanzos ${ }^{1}$ y María Montero-López Lena ${ }^{2}$
}

\begin{abstract}
Resumen
El objetivo del estudio consistió en obtener las características psicométricas de una escala de emociones compuestas hacia la conducta de cuidado del agua, desarrollada a partir de los supuestos de la teoría cognitiva de las emociones. Fueron analizadas las respuestas de una muestra intencional de 376 estudiantes universitarios de la Ciudad de México. Mediante un análisis factorial confirmatorio de segundo orden, con el método robusto de máxima verosimilitud, se ratificó la pertinencia de una agrupación final de tres dimensiones, alcanzando bondad de ajuste adecuada, y consistencia interna. La evidencia generada permite asumir que se cuenta, dentro del contexto mexicano, un instrumento válido y confiable que evalúa tres emociones específicas (gratitud, enojo y remordimiento) hacia acciones de consumo de agua.
\end{abstract}

Palabras clave: cuidado del agua, emociones, conducta pro-ambiental, psicología ambiental

\begin{abstract}
This study had the purpose of obtain the psychometric characteristics of a scale of composite emotions to the water conservation behavior, which was developed considering assumptions of cognitive theory of emotions. Responses from a purposive sample of 376 undergraduate students from Mexico City were analyzed. Through a second-order factor analysis, estimated by the robust maximum likelihood method, a final group of three dimensions was ratified, achieving accurate goodness of fit levels and internal consistency. The evidence generated allows for assume that there is, within the Mexican context, a valid and reliable instrument addressed to evaluate three specific emotions (gratitude, anger and remorse) towards actions of water consumption.
\end{abstract}

Keywords: water conservation, emotions, pro-environmental behavior, environmental psychology

Este artículo se derivó de un estudio apoyado parcialmente por el Consejo Nacional de Ciencia y Tecnología de México (CONACyT) mediante una beca (No. 223220) otorgada al primer autor para realizar estudios doctorales bajo la supervisión de la Dra. María Montero-López Lena.

${ }^{1}$ Doctor en Psicología. Facultad de Psicología, Universidad Nacional Autónoma de México. Facultad de Psicología, División de Estudios de Posgrado. Avenida Universidad 3004, Edificio D, segundo piso, cubículo 7, Colonia Copilco Universidad, Delegación Coyoacán, México. Tel.: (55)56222241. Correo: manriquezjuancarlos90@gmail.com

${ }^{2}$ Doctora en Psicología. Profesora de la Facultad de Psicología, Universidad Nacional Autónoma de México. Correo: monterol@unam.mx 


\section{Introducción}

Actualmente se reconoce que los problemas ecológicos repercuten en la calidad de vida y en el desarrollo de la humanidad. Si prevalecen las pautas de consumo, las tecnologías peligrosas para el ambiente y el crecimiento demográfico acelerado, las sociedades humanas actuales legarán a las generaciones futuras un planeta con recursos agotados (Schmuck \& Vlek, 2003).

En este sentido, los seres humanos poseen un rol dinámico para lograr un equilibrio entre los requerimientos del ambiente social y los del ambiente físico-químico. $\mathrm{Si}$ bien existen propuestas tecnológicas para reducir el deterioro ecológico (e.g. Clayton \& Myers, 2009; Steg \& Vlek, 2009), falta garantizar la apropiación cotidiana de éstas. Esta situación abre la posibilidad de que aportaciones derivadas de las ciencias sociales, incluida la psicología, permitan identificar variables críticas que reduzcan el impacto nocivo del quehacer humano sobre el entorno. En otras palabras, las soluciones para revertir la crisis ambiental, en cierta medida, se asocian con la identificación, la evaluación y la modificación de variables psicológicas y conductuales (Schmuck \& Vlek, 2003; Steg \& Vlek, 2009).

De los diferentes recursos naturales, el agua potable destaca por ser esencial para la existencia de vida en la Tierra (Syme, Porter, Goeft, y Kington, 2008). El Panel Intergubernamental sobre Cambio Climático (Intergovernmental Panel on Climate Change [IPCC por sus siglas en inglés], 2007) estimó que para el año 2030 cerca del $42 \%$ de la población mundial vivirá en países con agua potable insuficiente. Esta situación adquiere relevancia, ya que al existir escasez de este recurso las actividades humanas pueden quedar limitadas, mientras que los costos y las dificultades para su suministro doméstico se incrementan (Easter, 2009).

La escasez del agua potable se debe principalmente al consumo excesivo por parte de los seres humanos (Gifford, 2007). Por ello, parece razonable promover la investigación psicológica sobre los determinantes de esta conducta con el fin de fomentar el uso sustentable del líquido. Algunas de las variables identificadas como promotores del ahorro del agua son las creencias ambientales (Carrus, Bonnes, CorralVerdugo, Moser, \& Sinha, 2010), la perspectiva temporal hacia el futuro (Pinheiro \& CorralVerdugo, 2010), los motivos (Bustos, Flores, \& Andrade, 2002) y la percepción de autoeficacia (Palacios \& Bustos, 2013). Aunque existe escasa evidencia sobre factores emocionales vinculados a dicha conducta, existe mayor información sobre tales factores en relación con la conducta proambiental, la cual teóricamente se considera como un conjunto de diversas acciones que se realizan para el cuidado del ambiente y sus recursos (e.g. Carrus, Passafaro, \& Bonnes, 2008; Kals \& Maes, 2002; Tapia-Fonllem, CorralVerdugo, Gutiérrez-Sida, Mireles-Acosta, \& Tirado-Medina, 2010).

En opinión de Fernández-Abascal, Palmero y Martínez-Sánchez (2002), el constructo de emoción carece de límites precisos y quizá sea el proceso psicológico menos conocido. Es probable que tal situación haya causado que las emociones suelan confundirse con otros constructos, y que existan diversas clasificaciones sobre éstas. En razón de esto, autores como Fernández et al. (2002), y Rodríguez y Padilla (2008) especificaron algunos de los constructos afines a la emoción:

1. Emociones: son eventos discretos pasajeros o episódicos, con una meta específica, motivan alguna conducta derivada del objeto promotor de esa emoción en particular. Se relacionan con la adaptación de un organismo a condiciones ambientales específicas.

2. Afecto: incluye cualquier representación de valor (bondad-maldad, preferencias y actitudes [disposiciones afectivas] y emociones). Se relaciona con la valoración de las distintas situaciones a las que se enfrenta el individuo.

3. Estados de ánimo (o humor): es la suma de los componentes de expresiones subjetivas de las emociones básicas: felicidad, tristeza, miedo, disgusto, enojo, sorpresa, donde el objeto no es sobresaliente o es inespecífico. Tienen mayor duración y menor intensidad que una emoción.

Un caso que merece atención es la posible confusión entre las emociones y las actitudes, reconociéndose cierto grado de similitud entre estas variables, así como una distinción imprecisa de ambas (Bagozzi, Gopinath, \& Nyer, 1999). Para Ajzen y Fishbein (1980), la actitud consiste 
en una evaluación general individual de favorabilidad o desfavorabilidad hacia un objeto o conducta. Asimismo, Eagly y Chaiken (1993) supusieron que la actitud expresa una evaluación en grados de favor o desfavor. Las actitudes pueden ser apreciadas como juicios individuales acerca de si realizar una conducta dada es buena o mala, o que el individuo está a favor o en contra de llevar a cabo dicha conducta (Ajzen \& Fishbein, 1980, p. 56). No obstante, Ajzen y Fishbein aprecian a las actitudes como sistemas complejos que comprenden creencias individuales de tres categorías: cognitivas, afectivas y conductuales. El constructo de actitud fue incorporado en teorías (e.g. las de la acción razonada y de la acción planeada) para explicar la conducta de los individuos. Sin embargo, en opinión de Perugini y Bagozzi (2004), en el caso de la teoría de la acción planeada (y quizá también en la teoría de la acción razonada) el componente cognitivo evaluativo de la actitud ha sido enfatizado, relegando al afectivo.

Con objeto de distinguir a las actitudes, como juicios evaluativos, de las emociones, Bagozzi et al. (1999) expresaron la necesidad de tener en consideración algunos puntos:

1. Aunque ambas disposiciones refieren estados de preparación, la emoción posee mayor intensidad en contraste con la de las actitudes

2. Las actitudes, pese a surgir de cambios en eventos (como las emociones), no necesariamente implican la activación (arousal) presente en las emociones.

3. Mientras que las actitudes pueden almacenarse en largos periodos de tiempo y ser recuperadas, las emociones suelen consistir en estados de preparación en progreso

4. Las emociones se originan como respuesta ante algo relevante para el bienestar del individuo. Las evaluaciones específicas e individuales, y no los eventos o las circunstancias físicas per se, son las que producen emociones. Este punto es profundizado por las teorías de emociones de índole cognitivo.

5. Las emociones pueden estimular directamente el inicio de un acto. En cambio, las actitudes pueden requerir de motivación adicional que se realice una conducta.

Con base en este listado, se desprende que aunque la emoción y la actitud comparten ciertas características, no son constructos equivalentes. En este sentido, parece necesario tratar a estos términos como distintos, y por consiguiente evaluar de manera independiente sus efectos en relación a una conducta dada.

Como se señaló, se carece de un acuerdo generalizado respecto a cómo definir a la emoción, circunstancia que ha provocado la existencia de diversas categorías de emociones. $\mathrm{Al}$ hacer una revisión de estudios, Reidl (2005) mencionó que las definiciones de emoción son variadas por depender de los diversos marcos referenciales de los estudiosos que las abordan. De esta forma Reidl (op cit.) señala que, si los investigadores destacan aspectos biológicos para definir a la emoción tienden a admitir la existencia de emociones básicas. Dentro de esta vertiente, el rol de la evolución en la formación de los aspectos emocionales es resaltado, así como el valor adaptativo que algunas emociones (denominadas como básicas) desempeñan en el afrontamiento de tareas vitales fundamentales del organismo, como la reproducción o evitar amenazas a la supervivencia (e.g. Ekman, 1992; Plutchik, 2001). En el caso contrario, la postura cognitiva suele rechazar la existencia de emociones básicas, y por ende plantean que las emociones tienen su origen en la evaluación cognitiva que realiza el individuo ante una situación.

Otra manera de hacer una distinción entre emociones es tomando en cuenta su valencia. Las emociones permiten evaluar si los estímulos de una situación dada son significativos para lograr el bienestar y/o metas valoradas. Así, algunas funciones desempeñadas por las emociones son proporcionar de dirección a la atención, guiar la toma de decisiones, estimular el aprendizaje y motivar la conducta (Cacioppo, Gardner, \& Bernston, 1999), y facilitar comportamientos para resolver desafíos adaptativos (Bartlett \& DeSteno, 2006; Schmidt, 2008). En diversos estudios (e.g. Frijda, 1986; Lazarus, 1993; Lewis, 2008; Rodríguez \& Padilla, 2008) se alude a que las emociones dependen de una evaluación con la que se juzga si la transacción con los eventos ambientales es positiva o negativa para el organismo, lo cual determina la valencia emocional. Se hace referencia a emociones negativas cuando la transacción con el ambiente se percibe amenazante, dañina, o que obstaculiza, 
retrasa o imposibilita la satisfacción de necesidades, el logro de metas, compromisos o la realización de valores positivos. En sentido opuesto, si dicha transacción es evaluada como benigna, que no aporten indicios de que exista un problema a resolver, entonces se considera que las emociones son positivas (Bagozzi, 2000; Fredrickson \& Cohn, 2008; Frijda, 1986; Rodríguez \& Padilla, 2008). Cabe destacar que es pertinente tratar de manera independiente a los factores emocionales positivos de los negativos y no simplemente considerarlos como opuestos (Fredrickson \& Cohn, 2008).

Retomando la revisión de estudios psicoambientales, existe evidencia que documenta que algunas emociones negativas como la culpa, la vergüenza o la indignación, inhiben conductas destructoras del ambiente (Durán, Alzate, López, \& Sabucedo, 2007; Ferguson \& Branscombe, 2010; Kaiser, Schultz, Berenguer, CorralVerdugo, \& Tankha, 2008; Kals \& Maes, 2002; Tapia-Fonllem et al., 2010). En contraste, emociones positivas como la afinidad hacia la diversidad, la simpatía y la empatía se vinculan con tendencias proambientales (Berenguer, 2010; Bonnes, Carrus, Corral-Verdugo, \& Passafaro, 2010; Kals \& Maes, 2002; Kals \& Müller, 2012; Kals, Schumacher, \& Montada, 1999; Sevillano, Aragonés, \& Schultz, 2007; Tapia-Fonllem et al., 2010).

Aunque las investigaciones previas documentan el nexo entre las emociones y la conducta proambiental, rara vez se aborda su efecto hacia una conducta proambiental específica. Esta situación podría ser una limitante si se desea profundizar en el efecto que cada conducta proambiental particular recibe de los factores emocionales. Autores como McKenzieMohr, Nemiroff, Beers y Desmarais (1995) sugieren que se evalúen conductas específicas en vez de agruparlas como conducta proambiental, lo cual implicaría detectar cuáles son los factores más relevantes en cada conducta particular. Por otro lado, aunque se han documentado algunos factores emocionales vinculados con el impacto restaurativo que provocan los ambientes acuáticos (White, Smith, Humphryes, Pahl, Snelling, \& Depledge, 2010), extrañamente se carecía de estudios que vincularan a las emociones con el ahorro de agua. Poco después, de Miranda
Coelho, Gouveia, de Souza, Milfont y Barros (2016) desarrollaron una escala unidimensional para la Escala de Evaluación de las Emociones hacia el Derroche de Agua (EEEDA), encontrando una estructura unidimensional. El factor reportado, angustia por el derroche de agua, mostró mayor tendencia a evaluar emociones de valencia negativa, principalmente enojo.

Aunque la EEEDA puede ser útil en evaluar el impacto de factores emocionales en el derroche del agua, parece plausible que más de una emoción ocurra cuando se evalúan las acciones de consumo de agua, ya sea de derroche o de ahorro del recurso. Mediante el empleo de una aproximación que discrimine emociones en función de los antecedentes y las consecuencias asociadas a una conducta determinada, puede facilitarse la vinculación de emociones concretas con conductas especificas (Kaiser et al., 2008).

La teoría cognitiva de las emociones (Ortony, Clore, \& Collins, 1988/1996) plantea que la cognición contribuye a la existencia de distintas emociones en función de la codificación específica que realizan los individuos al evaluar una situación específica. El término appraisal, (traducido como evaluación), es definido por Moors, Ellsworth, Scherer, \& Frijda (2013) como un proceso que detecta y valora la significancia del ambiente para el bienestar. El que las personas respondan con distintas emociones a la misma situación depende de cómo la interpreten o la evalúen, supuesto esencial de las teorías de emociones de evaluación cognitiva. Así, es la evaluación de una situación y no la situación per se, lo que determina la cualidad e intensidad de una respuesta emocional (Lazarus, 1993; Siemer, Mauss, \& Gross, 2007). Para Scherer (2004), las teorías de evaluación cognitiva plantean que la naturaleza e intensidad de las emociones están determinadas principalmente por la evaluación subjetiva del significado y consecuencias de un evento para el individuo.

En la teoría cognitiva de las emociones (Ortony et al., 1988/1996), las emociones son representadas como un conjunto de reacciones distintas y basadas en orígenes cognitivos, ya que existen condiciones desencadenantes específicas para cada emoción. Así, las emociones consisten en "reacciones con valencia ante acontecimientos, agentes u objetos, cuya naturaleza particular está 
determinada por la manera en cómo se interpreta la situación desencadenante" (Ortony et al., op cit., p. 16). La teoría cognitiva de las emociones plantea tres grupos generales de emociones: a) de bienestar o dirigidas a acontecimientos, con énfasis en las consecuencias, b) de atribución o dirigidas a las acciones de agentes (el propio individuo u otras personas), y búsqueda de aprobación, c) dirigidas a objetos, en las que se evalúa la inclinación hacia propiedades de los objetos.

Además de los tres grupos principales, Ortony et al. (op cit) identificaron un conjunto adicional de emociones, vinculadas al bienestar y a la atribución simultáneamente, llamadas emociones compuestas. Estos autores las definen en razón de que es un grupo de emociones que "surgen al tomar en consideración simultáneamente tanto la acción de un agente como el acontecimiento resultante y sus consecuencias [...] implican más que la mera ocurrencia simultánea de las correspondientes emociones que las constituyen" (Ortony et al., op cit., p. 30). Sobre las condiciones que las desencadenan, dichos autores señalaron que a) la deseabilidad alude a si un acontecimiento es deseable o indeseable, y b) respecto al agente que ha ocasionado con sus acciones tal evento puede tratarse del individuo mismo u otras personas. Para Ortony et al. (op cit.) las emociones compuestas se encuentran unificadas y sus elementos constituyentes no son experimentados independientemente, e incluso reconocen la posibilidad de que posean una mayor intensidad que las emociones en que están basadas, por provenir de dos fuentes. De esta manera, el enojo, la gratitud, el remordimiento y la complacencia son incluidas dentro de este conjunto de emociones.

Un individuo experimenta gratitud si considera que las acciones de otras personas son favorables y conducen a resultados deseables. La gratitud incrementa las tendencias altruistas y motiva la conducta recíproca futura (Bartlett \& DeSteno, 2006; Emmons \& Mishra, 2010; Schmidt, 2008). Diversos estudios (Lambert, Fincham, Stillman, \& Dean, 2009; ManríquezBetanzos, Corral-Verdugo, Vanegas-Rico, TapiaFonllem, \& Fraijo-Sing, 2016; Naito et al., 2010; Polak \& McCullough, 2006) reportan que tendencias proambientales se relacionan con la gratitud.

El enojo resulta de atribuir a otras personas las consecuencias indeseables de acciones concretas. En el campo psicoambiental, el enojo muestra similitud a los sentimientos de indignación ante acciones antiecológicas de otras personas (Durán et al., 2007; Kals \& Maes, 2002; Tapia-Fonllem et al., 2010).

En cuanto al remordimiento, se trata de una emoción afín a la culpa, dirigida a reparar o evitar un fracaso o falla mediante conductas correctivas (Lewis, 2008). Dicha emoción inhibe tendencias destructivas hacia el ambiente (Ferguson y Branscombe, 2010; Harth, Leach, \& Kessler, 2013; Kaiser et al., 2008).

Finalmente, la complacencia refiere un estado en el que una persona juzga que sus acciones son exitosas para lograr un resultado deseado. Puede vincularse a lo hedónico, entendido como la búsqueda de placer (e.g. Huta, 2013). Es posible que favorezca la tendencia a la gratificación inmediata, y por lo tanto que conduzca a un alto consumo de agua (Iwata, 2001).

A partir de la propuesta teórica de Ortony et al. (1988/1996) es factible plantear cuatro postulados en relación al consumo de agua: a) esta conducta puede generar diferentes emociones, dependiendo cómo sea evaluado, b) puede tener diferentes grados de deseabilidad, en razón de las consecuencias a las que puede conducir, c) es provocado por acciones humanas susceptibles de aprobación, ya que se puede evaluar el propio consumo de agua, o bien el que realizan otras personas y d) se carece de algún instrumento con buenas propiedades psicométricas que documenten emociones vinculadas a esta conducta. Las acciones de uno mismo u otras personas pueden juzgarse como adecuadas o inadecuadas dependiendo si se considera que el consumo de agua, bajo o alto, resulta en consecuencias benéficas o perjudiciales (e.g. seguir contando con el recurso, o afrontar la carencia de éste). Para sufragar la ausencia de una medida que evalúe emociones hacia el consumo del agua, se llevó a cabo un estudio orientado a obtener características psicométricas de un instrumento sobre las emociones compuestas de gratitud, enojo, remordimiento y complacencia hacia dicha conducta. 


\section{Método}

\section{Participantes}

Se efectuó un estudio de campo exploratorio, con un diseño transeccional de grupos independientes (Kerlinger \& Lee, 2002). Los participantes fueron en total 376 estudiantes de una universidad pública de la Ciudad de México, 225 mujeres y 151 varones, con edades entre los 17 y 30 años $(M=20.14, D E=2.01)$.

\section{Instrumento}

Se generó un cuestionario que inicialmente contenía 40 reactivos, desarrollados con base en la teoría cognitiva de las emociones (Ortony et al., 1988/1996). Posee formato Likert de cinco opciones de respuesta, indicando la frecuencia (desde "nunca" a "frecuentemente") con el participante experimenta gratitud, enojo, complacencia y remordimiento respecto a acciones de consumo de agua individuales y de otras personas. Los cuatro factores iniciales fueron definidos conceptualmente de la siguiente manera

1. La Gratitud evalúa el agrado que se manifestaba por acciones efectuadas por otras personas al cuidar del líquido (e.g. "aprecio a las personas que enseñan a otras como cuidar el agua"). Contó con 25 reactivos.

2. El Enojo se vincula con la desaprobación de acciones de otras personas y el descontento por el alto consumo de agua, (e.g. "me irrita que mis familiares desperdicien el agua que otros necesitan"), con 27 reactivos.

3. La Complacencia integra la aprobación de acciones individuales y el gozo por un acontecimiento deseable relacionado con el alto consumo de agua (e.g. "me gusta utilizar bastante agua cuando lavo los platos"), con 13 reactivos.

4. El Remordimiento alude la desaprobación de acciones propias y el descontento por un acontecimiento indeseable asociado con el alto consumo de agua, (e.g. "siento remordimiento cuando desperdicio agua en mi casa"). Contó con 12 reactivos.

\section{Procedimiento}

El cuestionario se aplicó en diversas facultades universitarias, donde los estudiantes acuden regularmente a clases. Se contactó con profesores de grupos, quienes permitieron la aplicación del cuestionario a sus alumnos. Se les informó sobre el propósito del estudio y los estudiantes participaron de manera voluntaria y gratuita en el estudio. La aplicación duró 15 minutos aproximadamente. Después de agradecer la colaboración de los participantes, se procedió al análisis estadístico de los datos, para lo cual se emplearon tanto el SPSS 21 como el EQS 6.1

\section{Análisis de Datos}

En una primera etapa se efectuaron análisis factoriales confirmatorios (AFC) por escala para obtener evidencia de validez de constructo de los factores a prueba. Autores como Kline (2005) y Nunnally y Bernstein (1995) mencionan la obtención de validez convergente si existen pesos factoriales altos y significativos entre las variables observadas y los factores propuestos. Para elegir el método de estimación del modelo propuesto, se observó si los datos cumplían el supuesto de distribución normal, lo cual es indicado por el coeficiente normalizado multivariante de Mardia: valores mayores a siete indican una distribución no normal (West, Curran, \& Fincham, 1995). Ante esto, se sugiere emplear el método robusto de máxima verosimilitud (Satorra y Bentler, 2001). El ajuste de las estructuras factoriales se evaluó a través de seis indicadores. La $\chi^{2}$, que evalúa la discrepancia entre la matriz de datos con el modelo propuesto, de la cual se esperó un valor bajo y no significativo $(p<.05)$. Además, fueron retomados los índices prácticos de ajuste: CFI (índice de ajuste comparativo), NNFI (índice de ajuste no normalizado) y RMSEA (raíz del residuo cuadrático promedio de aproximación). Para los primeros dos índices, se esperaba que su valor fuese $\geq .95$ para indicar ajuste del modelo. En el caso del RMSEA, el ajuste se obtiene si el valor obtenido es $\leq .05$. Por último, se tomó en cuenta el intervalo de confianza de $90 \%$ del RMSEA, del que se espera tenga su límite superior en un valor <.10 (Byrne, 2006; Kline, 2005).

Posteriormente se obtuvieron estadísticas descriptivas y el coeficiente alfa de Cronbach para cada escala. También se empleó la prueba de correlación bivariada de Pearson, para evidenciar la relación entre los tres factores, lo que podría conducir a la prueba de un AFC con un factor de segundo orden. 
Tabla 1. Estadísticas univariadas y confiabilidad de las medidas empleadas

\begin{tabular}{|c|c|c|c|c|c|c|c|}
\hline Subescala/ Reactivos & $N$ & Min & Max & Media & $D T$ & Asimetría & Curtosis \\
\hline \multicolumn{8}{|l|}{$\begin{array}{l}\text { Enojo ante el derroche del agua } \\
(\text { Alfa=.89) }\end{array}$} \\
\hline $\begin{array}{l}\text { Me irrita saber que existe gente que desperdicia agua sin } \\
\text { pensar en otras personas }\end{array}$ & 376 & 1 & 5 & 4.20 & 0.88 & -1.11 & 1.06 \\
\hline Me enfurece ver a otras personas desperdiciando agua & 375 & 1 & 5 & 4.13 & 1.04 & -1.11 & 0.60 \\
\hline $\begin{array}{l}\text { Siento enojo cuando otras personas desperdician el agua } \\
\text { que les falta a otros }\end{array}$ & 376 & 1 & 5 & 4.20 & 0.96 & -1.21 & 1.13 \\
\hline $\begin{array}{l}\text { Me enfurece saber que a algunas personas les importe } \\
\text { poco que el agua se acabe }\end{array}$ & 374 & 1 & 5 & 4.06 & 1.05 & -1.06 & 0.57 \\
\hline $\begin{array}{l}\text { Me irrita que mis familiares desperdicien el agua que } \\
\text { otros necesitan }\end{array}$ & 376 & 1 & 5 & 3.95 & 1.01 & -0.77 & 0.01 \\
\hline $\begin{array}{l}\text { Me siento indignado cuando la gente desperdicia agua } \\
\text { en su casa }\end{array}$ & 371 & 1 & 5 & 4.02 & 0.97 & -0.85 & 0.28 \\
\hline \multicolumn{8}{|l|}{$\begin{array}{l}\text { Gratitud hacia el } \\
\text { cuidado del agua }(\mathrm{Alfa}=.90)\end{array}$} \\
\hline $\begin{array}{l}\text { Aprecio a las personas que enseñan a otras como cuidar } \\
\text { el agua }\end{array}$ & 374 & 1 & 5 & 4.18 & 0.90 & -1.09 & 1.11 \\
\hline $\begin{array}{l}\text { Siento aprecio por otras personas cuando evitan que se } \\
\text { desperdicie el agua en sus casas }\end{array}$ & 373 & 1 & 5 & 3.96 & 0.94 & -0.68 & -0.07 \\
\hline $\begin{array}{l}\text { Agradezco a mi familia cuando reparan una fuga en mi } \\
\text { casa }\end{array}$ & 376 & 1 & 5 & 4.07 & 0.97 & -0.96 & 0.50 \\
\hline $\begin{array}{l}\text { Agradezco que las personas cuiden del agua no } \\
\text { solamente por lo que cuesta }\end{array}$ & 376 & 1 & 5 & 4.13 & 0.88 & -0.96 & 0.76 \\
\hline $\begin{array}{l}\text { Aprecio a las personas que se esfuerzan para proteger el } \\
\text { agua }\end{array}$ & 375 & 1 & 5 & 4.14 & 0.92 & -1.09 & 0.94 \\
\hline \multicolumn{8}{|l|}{ Subescala/ Reactivos } \\
\hline $\begin{array}{l}\text { Agradezco que algunas personas hablen sobre lo } \\
\text { importante que es cuidar el agua }\end{array}$ & 376 & 1 & 5 & 4.05 & 0.89 & -0.80 & 0.32 \\
\hline Aprecio que mi familia se interese en cómo ahorrar agua & 369 & 1 & 5 & 4.08 & 0.90 & -0.96 & 0.82 \\
\hline $\begin{array}{l}\text { Siento agradecimiento por los vecinos que cuidan del } \\
\text { agua en la colonia }\end{array}$ & 376 & 1 & 5 & 3.89 & 1.04 & -0.87 & 0.31 \\
\hline \multicolumn{8}{|l|}{$\begin{array}{l}\text { Remordimiento hacia el } \\
\text { Derroche del agua }(\text { alfa }=.84)\end{array}$} \\
\hline $\begin{array}{l}\text { Me siento culpable cuando mis acciones provocan que } \\
\text { les falte agua a otras personas }\end{array}$ & 376 & 1 & 5 & 3.33 & 1.24 & -0.26 & $\begin{array}{l}-0.93 \\
\end{array}$ \\
\hline $\begin{array}{l}\text { Me enojo conmigo mismo cuando dejo la llave abierta } \\
\text { mucho tiempo }\end{array}$ & 372 & 1 & 5 & 3.44 & 1.32 & -0.39 & -1.0 \\
\hline $\begin{array}{l}\text { Me enojaría conmigo mismo si hoy desperdicio el agua } \\
\text { que mis hijos podrían usar en el futuro }\end{array}$ & 374 & 1 & 5 & 3.90 & 1.11 & -0.82 & -0.14 \\
\hline $\begin{array}{l}\text { Me enojo conmigo mismo cuando gasto agua y luego me } \\
\text { falta para otras actividades }\end{array}$ & 375 & 1 & 5 & 3.64 & 1.10 & -0.59 & -0.56 \\
\hline $\begin{array}{l}\text { Tengo remordimiento de haber olvidado cerrar bien } \\
\text { alguna llave del agua en casa }\end{array}$ & 375 & 1 & 5 & 3.62 & 1.17 & -0.55 & -0.46 \\
\hline $\begin{array}{l}\text { Siento remordimiento cuando desperdicio agua en } \mathrm{mi} \\
\text { casa }\end{array}$ & 373 & 1 & 5 & 3.73 & 1.09 & -0.62 & -0.26 \\
\hline
\end{tabular}

Finalmente, para probar este último supuesto, se efectuó un AFC que integraba a los tres factores, para probar si una estructura multifactorial mostraba ajuste con los datos obtenidos.

\section{Resultados}

La Tabla 1 exhibe estadísticas univariadas (medias, desviaciones estándar, valores máximos y mínimos, asimetría, curtosis) y el coeficiente alfa de Cronbach de las escalas empleadas. Las medidas alcanzaron valores de alfa $\geq .60$, exceptuando los reactivos relativos a la emoción de complacencia, por lo que fueron excluidos de análisis posteriores.

Se obtuvieron correlaciones bivariadas para probar la existencia de relaciones significativas entre las dimensiones obtenidas. Como se aprecia en la Tabla 2, las relaciones entre los tres factores fueron altas y significativas, evidenciando consistencia entre los datos obtenidos y la teoría retomada. Se planteó la posibilidad de que los tres 


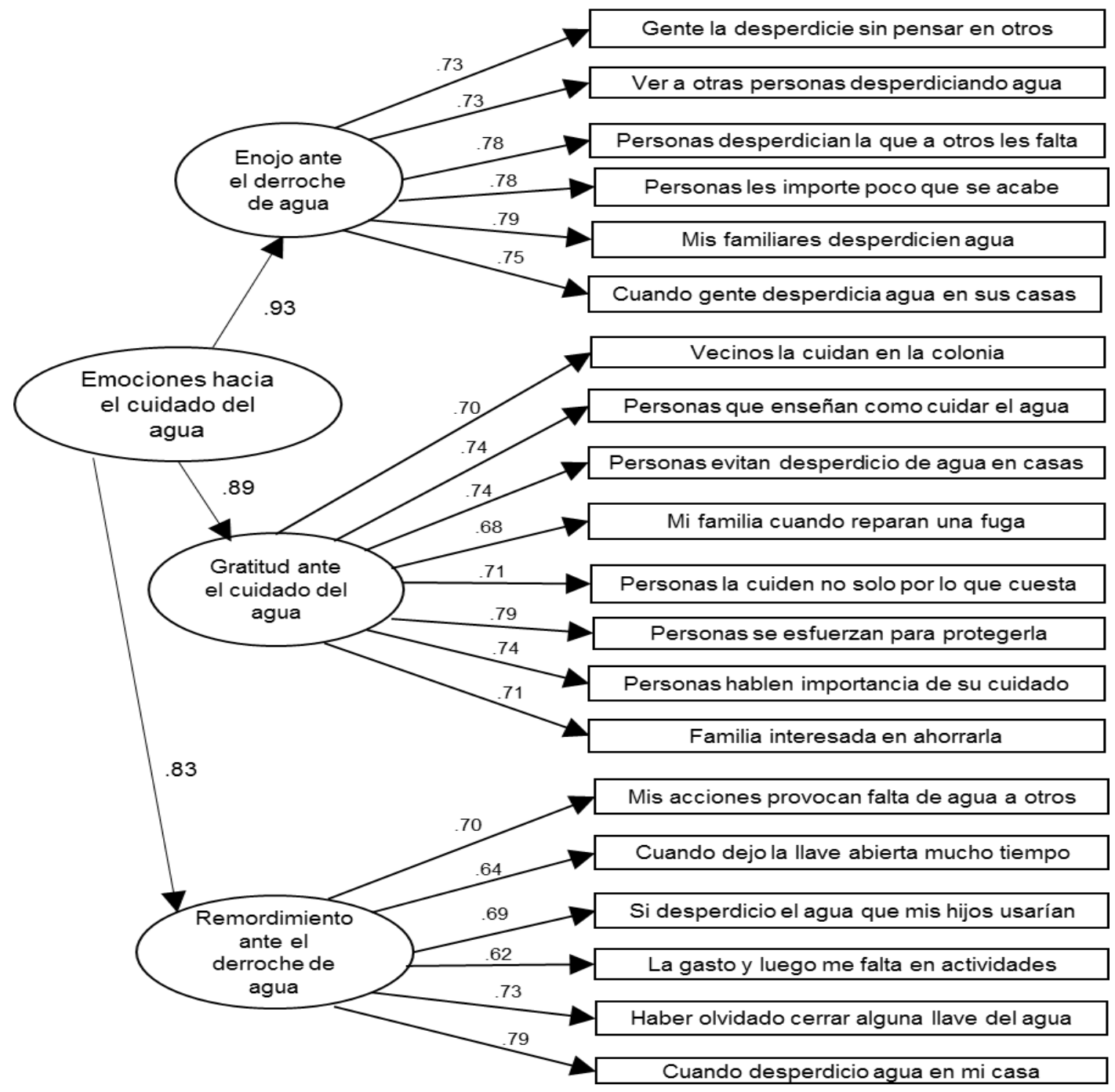

Figura 1. Análisis Factorial Confirmatorio de la Escala de Emociones hacia el Cuidado del Agua. Pesos factoriales significativos $(t>1.96, p<.05)$. Bondad de ajuste: $\mathrm{S}-\mathrm{B} \chi^{2}(167 \mathrm{~g} .1 ., \mathrm{N}=376)=275.59, p>.05 ; C F I=.96 ; N N F I=.95$; RMSEA=.04; Intervalo de Confianza del 90\% del RMSEA (.03, .05).

Tabla 2. Correlaciones entre los factores de la escala

\begin{tabular}{lccc}
\hline & 1 & 2 & 3 \\
\hline 1 Gratitud & 1.0 & & \\
2 Enojo & $0.78^{* *}$ & 1.0 & \\
3 Remordimiento & $0.63^{* *}$ & $0.66^{* *}$ & 1.0 \\
\hline$*^{* *} p<.01$ (bilateral) & & &
\end{tabular}

factores exhibieran dependencia hacia un factor de orden superior. En vista de lo anterior se consideró pertinente emplear un AFC de segundo orden.

Finalmente, en la Figura 1 se muestra la estructura sometida a prueba. El valor del coeficiente normalizado multivariante de Mardia se situó en 58.73 , por lo que se optó por la estimación del modelo con el método robusto de máxima verosimilitud. $\mathrm{Si}$ bien el valor de Chi cuadrada escalada de Satorra-Bentler fue significativo $\left(\mathrm{S}-\mathrm{B} \quad \chi^{2} \quad[167, \quad \mathrm{~N}=360]=275.59\right.$, $p<.01)$, los índices $C F I$ y $N N F I$ se situaron en .95 y .96, respectivamente. El RMSEA presentó un valor de .04 , mientras que el intervalo de confianza de $90 \%$ de RMSEA exhibió valores adecuados $(.03, .05)$. Todos los pesos factoriales fueron mayores a .60 , y significativos $(t>1.96$, $p<.05)$, lo que significa que existen relaciones fuertes entre el factor y sus indicadores. Estos datos permiten asumir que el modelo obtenido presenta un buen ajuste con los datos obtenidos. Las variables latentes de gratitud, enojo y remordimiento mostraron dependencia estadística hacia el factor de segundo orden denominado Emociones hacia el consumo del agua, con lo que se avala la estructura jerárquica hipotetizada. 


\section{Discusión}

El objetivo de este trabajo fue obtener las características psicométricas de un instrumento sobre emociones compuestas dirigidas hacia el consumo del agua. Los resultados indican que la complacencia ante el derroche de agua no pudo ratificarse mediante el empleo del AFC, quedando tres emociones: gratitud hacia el cuidado del agua, enojo y remordimiento dirigidos hacia el derroche de dicho recurso.

La evidencia indica que estas emociones, dos con valencia negativa y una con valencia positiva, poseen alta covariación. Asimismo, se estipuló que un factor de segundo orden, denominado emociones hacia el consumo de agua, mostraría cargas factoriales de los tres factores de primer orden que denotan a las tres emociones abordadas. No obstante, en razón de que dichas cargas factoriales fueron altas, parece viable suponer que si un individuo puntúa alto en una emoción particular, también lo haga de en las emociones restantes. No obstante, una explicación alternativa consiste en que existe una estructura unidimensional sobre emociones. Dicho resultado, aunque inesperado, guarda relación con la estructura EEEDA (de Miranda Coelho et al., 2016).

Pese a los resultados obtenidos, y considerando su nivel de consistencia interna, parece ser posible que las subescalas sean retomadas individualmente en estudios futuros. Dicha sugerencia está basada en el supuesto de que cada emoción resulta de distintas interpretaciones, como mencionan Ortony et al. (op. cit.), pero sobre todo, que cada una de ellas puede conducir a resultados de diferente naturaleza. Aunque las emociones negativas pueden ser útiles a corto plazo, porque advierten de un problema a resolverse a la brevedad posible, emociones positivas pueden tener efectos a largo plazo (de Miranda Coelho et al., 2016; Fredrickson \& Cohn, 2008). Vale la pena destacar que las emociones dirigidas hacia las acciones de otras personas (gratitud y enojo), pueden fomentar la participación colectiva para cuidar del agua, pero quizá disminuyan el involucramiento personal en la conducta. Por otro lado, parece posible que el remordimiento menoscabe otros recursos psicológicos, por lo que tal vez pudiese ser menos relevante en la conservación del agua. No obstante se requieren de estudios futuros para probar dichas suposiciones.

Otro punto a mencionar se relaciona con el rol de la complacencia ante el consumo de agua. En la presente investigación la complacencia se dirigió hacia el derroche de agua, destacando el componente hedónico de dicha conducta (e.g. Huta, 2013). Sin embargo, es posible que los participantes hayan sesgado sus respuestas al subestimar el alto consumo de agua que realizan, ya que el derroche del líquido se desacredita socialmente. Por otro lado, se reconoce que esta emoción podría resultar de ahorrar agua individualmente. En otras palabras, es posible que la complacencia posea dos direcciones: una enfocada hacia una percepción positiva de derrochar agua, utilizando dicho recurso para obtener placer y satisfacer deseos (Iwata, 2001), mientras que la segunda surja de evaluar al ahorro del líquido como un acto con consecuencias deseables (De Young, 2000). Aparentemente, tal contradicción entre ambas trayectorias influye en la falta de identificación factorial de esta emoción, siendo conveniente conocer qué consecuencias valora la gente cuando consume agua, ya que se podrían evaluar ambos tipos de complacencia dirigidos al consumo del agua.

La gratitud ha sido poco explorada en relación con la conducta proecológica, y parece pertinente integrarla al estudio sobre factores contribuyentes del ahorro del agua (ManríquezBetanzos et al., 2016). Esta sugerencia es formulada considerando que esta emoción disminuye los efectos negativos del materialismo e incrementa la satisfacción propia con la vida (Emmons \& Mishra, 2010; Lambert et al., 2009; Naito et al., 2010; Polak \& McCullough, 2006). Si una persona experimenta gratitud ante las acciones sociales de cuidado del agua es probable que se involucre en dicha conducta al seguir el modelo de otros. Parece probable que la gratitud contribuye a reforzar la cohesión social, aunque tal suposición requiere de evidencia empírica. Esta emoción puede ser importante a considerar en estudios psicoambientales, puesto que hay evidencia indirecta que señala consistentemente que las tendencias prosociales están relacionadas positivamente con las proambientales. 
En resumen, con base en los hallazgos de este estudio, es posible afirmar que el instrumento desarrollado exhibe congruencia con la teoría cognitiva de las emociones (Ortony et al., 1988/1996). Los hallazgos encontrados son consistentes con estudios previos (de Miranda Coelho, et al., 2016; Durán et al., 2007; Ferguson \& Branscombe, 2010; Harth et al., 2013; Kaiser et al., 2008; Kals \& Maes, 2002; Tapia et al., 2010) donde se ha documentado que el enojo y el remordimiento son afines a la indignación y a la culpa. Sobre la gratitud, se destaca su incorporación al estudio del consumo del agua, ya que se trata de una emoción positiva novedosa. Aunque la mayoría de los estudios sobre emociones enfocan las de valencia negativa (porque promueven respuestas a amenazas inmediatas), existe mayor apertura en reconocer que las emociones positivas influyen sobre diversos procesos cognitivos, sociales $\mathrm{y}$ biológicos (Fredrickson \& Cohn, 2008). Las emociones de enojo, remordimiento y gratitud permiten evaluar a la conducta de consumo de agua tanto a nivel individual como grupal, ya que la gratitud y el enojo resultan de evaluar acciones de otras personas, mientras que el remordimiento enfoca las acciones propias.

En estudios posteriores sería conveniente emplear una muestra con mayor representatividad o de mayor rango de edad, ya que los estudiantes universitarios no son representativos de la población en general. Sería pertinente verificar si la estructura factorial encontrada se mantiene al estudiar diferentes muestras de población abierta (e.g. amas de casa, adolescentes, jefes de familia) mediante una validación cruzada. No obstante, es relevante conocer el consumo de agua que efectúan los jóvenes, puesto que están en una etapa previa a asumir obligaciones, pero también poseen capacidades y aspiraciones que posiblemente se fijarán en la edad adulta. Cabe hacer mención que, en el futuro, muchos de estos jóvenes podrían desempeñarse como los futuros tomadores de decisiones en relación al uso que se le dé al agua, así como de otros recursos vitales.

Una recomendación de este estudio es la adición de los factores emocionales en modelos cognitivos sobre acciones proambientales, específicamente en los que enfocan al consumo de agua. Esta acción permitiría enriquecer la comprensión de esta conducta, al brindar una visión más completa sobre los determinantes psicológicos de dicha conducta. Un modelo cognitivo de emociones puede conducir a distintas ventajas en el campo psicoambiental, como explicar reacciones personales desiguales hacia un mismo evento, analizar componentes cognitivos de las emociones con más profundidad, generar intervenciones sobre componentes específicos de tales emociones (Kals \& Müller, 2012).

Los resultados obtenidos permiten asumir que ahora se cuenta con un instrumento válido y confiable para ser usado con poblaciones latinas semejantes a la empleada en este estudio. La escala aquí presentada da apoyo empírico a la propuesta de Ortony et al. (1988/1996), y sienta las bases para desarrollar instrumentos indirectos que consideren las emociones vinculadas con conductas específicas de consumo de recursos naturales.

\section{Referencias}

Ajzen, I., \& Fishbein, M. (1980). Understanding attitudes and predicting social behavior. EE.UU.: Prentice-Hall.

Bagozzi, R. P. (2000). The poverty of economic explanations of consumption and an action theory alternative. Managerial and Decision Economics, 21, 95-109.

Bagozzi, R. P., Gopinath, M., \& Nyer, P. U. (1999). The role of emotions in marketing. Journal of the Academy of Marketing Science, 27 (2), 184-206.

Bartlett, L., \& DeSteno, D. (2006). Gratitude and prosocial behavior: helping when it costs you. Psychological Science, 17, 319-325.

Berenguer, J. (2010). The effect of empathy in environmental moral reasoning. Environment and Behavior, 42, 110-134.

Bonnes, M., Carrus, G., Corral-Verdugo, V., \& Passafaro, P. (2010). The socio-psychological affinity towards diversity: From biodiversity to socio-ecological sustainability. En V. Corral-Verdugo, C. García-Cadena y M. Frías-Armenta (Eds.), Psychological Approaches to Sustainability: Current trends in theory, research and applications (pp. 125140). Nueva York, EE. UU.: Nova Science Publishers. 
Bustos, J. M., Flores, L. M., \& Andrade, P. (2004). Predicción de la conservación de agua a partir de factores socio-cognitivos. Medio Ambiente y Comportamiento Humano, 5, 5770.

Byrne, B. (2006). Structural Equation Modeling with EQS. Basic concepts, applications, and programming. ( $2^{\mathrm{a}}$. ed.). New Jersey, USA: Lawrence Erlbaum Associates.

Cacioppo, J., Gardner, W., \& Bernston, G. (1999). The affect system has parallel and integrative processing components: Form follows function. Journal of Personality and Social Psychology, 76, 839-855.

Carrus, G., Passafaro, P., \& Bonnes, M. (2008). Emotions, habits and rational choices in ecological behaviours: The case of recycling and use of public transportation. Journal of Environmental Psychology, 28, 51-62.

Carrus, G., Bonnes, M., Corral-Verdugo, V., Moser, G., \& Sinha, J. (2010). Socialpsychological and contextual predictors of sustainable water consumption. En V. CorralVerdugo, C., García-Cadena, y M. FríasArmenta (Eds.). Psychological Approaches to Sustainability: current trends in Theory, Research and Applications (pp. 43-60). Nueva York, EE.UU.: Nova Science Publishers.

Clayton, S., \& Myers, G. (2009). Conservation Psychology. Understanding and promoting human care for nature. United Kingdom: Wiley-Blackwell.

de Miranda Coelho, J. A. P., Gouveia, V. V., de Souza, G. H. S., Milfont, T. L., \& Barros, B. N. R. (2016). Emotions toward water consumption: Conservation and wastage. Revista Latinoamericana de Psicología, 48 (2), 117-126.

De Young, R. (2000). Expanding and evaluating motives for environmentally responsible behavior. Journal of Social Issues, 56, 509526.

Durán, M., Alzate, M., López, W., \& Sabucedo, J. (2007). Emociones y comportamiento proambiental. Revista Latinoamericana de Psicología, 39, 287-296.

Eagly, A. H., \& Chaiken, S. (1993). The Psychology of Attitudes. Fort Worth, Texas: Harcourt Brace Jovanovich.
Easter, W. (2009). Demand management, privatization, water markets, and efficient water allocation in our cities. En L. A. Baker (Ed.). The Water Environment of Cities (pp. 259-274). EE. UU.: Springer.

Ekman, P. (1992). An argument for basic emotions. Cognition \& Emotion, 6 (3/4), 169200.

Emmons, R., \& Mishra, A. (2010). Why gratitude enhances well-being: what we know, what we need to know. En K. Sheldon, T. Kashdan, y M.Steger (Eds.). Designing positive psychology. Taking stock and moving forward. (248-262). New York: Oxford University Press.

Ferguson, M., \& Branscombe, N. (2010). Collective guilt mediates the effect of beliefs about global warming on willingness to engage in mitigation behavior. Journal of Environmental Psychology, 30, 135-142.

Fernández-Abascal, E. G., Palmero, F., \& Martínez-Sánchez, F. (2002). Introducción a la psicología de la motivación y la emoción. En F. Palmero, E. G. Fernández-Abascal, F. Martínez y M. Chóliz, (Coords.). Psicología de la Motivación y la Emoción (pp. 3-34). Madrid, España: Mc Graw Hill / Interamericana.

Fredrickson, B., \& Cohn, M. (2008). Positive emotions. En M. Lewis, J. Haviland-Jones y L. Feldman (Eds.). Handbook of Emotions. (3a. ed., pp. 777-796). Nueva York, EE.UU.: The Guilford Press.

Fridja, N. (1986). The Emotions. Londres, Reino Unido: Cambridge University Press.

Gifford, R. (2007). Environmental Psychology. Principles and practice. Canadá: Optimal Books.

Harth, N., Leach, C., \& Kessler, T. (2013). Guilt, anger and pride about in-group environmental behavior: Different emotions predict distinct intentions. Journal of Environmental Psychology, 34, 18-26.

Huta, V. (2013). Eudaimonia. En S. David, I. Boniwell, y A.C. Ayers (Eds.), Oxford Handbook of Happiness (pp. 201-213). Oxford, Reino Unido: Oxford University Press.

Intergovernmental Panel on Climate Change [IPCC]. (2007). Climate Change 2007: 
Synthesis Report. R. Pachauri \& A. Reisinger (Eds.). IPCC: Geneva, Switzerland.

Iwata, O. (2001). Attitudinal determinants of environmentally responsible behavior. Social behavior and personality, 29, 183-190.

Kaiser, F. G., Schultz, P. W., Berenguer, J., Corral-Verdugo, V., \& Tankha, G. (2008). Extending planned environmentalism. Anticipated guilt and embarrassment across cultures. European Psychologist, 13, 288-297.

Kals, E., \& Maes, J. (2002). Sustainable development and emotions. En P. Schmuck y P. W. Schultz (Eds.), Psychology of Sustainable Development (pp. 97-122). Massachussetts, EE.UU.: Kluwer.

Kals, E., \& Müller, M. (2012). Emotions and Environment. En S. Clayton (Ed.) The Oxford Handbook of Environmental and Conservation Psychology. (pp. 128-147). Nueva York, EE.UU.: Oxford University Press.

Kals, E., Schumacher, D., \& Montada, L. (1999). Emotional affinity toward nature as a motivational basis to protect nature. Environment and Behavior, 31, 178-202.

Kline, R. (2005). Principles and practice of structural equation modelling. Second Edition. New York: The Guilford Press.

Lambert, N., Fincham, F., Stillman, T., \& Dean, L. (2009). More gratitude, less materialism: The mediating role of life satisfaction. The Journal of Positive Psychology, 4, 32-42.

Lazarus, R. S. (1993). From psychological stress to the emotions: A history of changing outlooks. Annual Review of Psychology, 44 (1), 1-22.

Lewis, M. (2008). Self-conscious emotions. Embarrassment, pride, shame, and guilt. En M. Lewis, J. Haviland-Jones, y L. Feldman (Eds.), Handbook of Emotions (3 ${ }^{\mathrm{a}} \mathrm{ed}$, pp. 742756). New York, USA: The Guilford Press.

Manríquez-Betanzos, J. C., Corral-Verdugo, V., Vanegas-Rico, M. C., Fraijo-Sing, B. S., \& Tapia-Fonllem, C. O. (2016). Positive (gratitude, eudaimonia) and negative (scarcity, costs) determinants of wáter conservation behaviour / Determinantes positivos (gratitud, eudaimonia) y negativos (escasez, costos) del ahorro de agua. Psyecology, 7 (2), 178-200.
McKenzie-Mohr, D., Nemiroff, S., Beers, L., \& Desmarais, S. (1995). Determinants of responsible environmental behaviour. Journal of Social Issues, 54 (4), 139-156.

Moors, A., Ellsworth, P., Scherer, K. R., \& Frijda, N. (2013). Appraisal theories of emotion: State of the art and future development. Emotion Review, 5 (2), 119-124.

Naito, T., Matsuda, T., Intasuwan, P., Chuawanlee, W., Thanachanan, S., Ounthitiwat, J., \& Fukushima, M. (2010). Gratitude for, and regret toward, nature: Relationships to environmental intent of university students from Japan. Social Behavior and Personality, 38, 993-1008.

Nunnally, J., \& Bernstein, I. (1995). Teoría Psicométrica. ( $3^{\mathrm{a}}$. ed.) México: McGraw-Hill.

Ortony, A., Clore, G., \& Collins, A. (1996). La estructura cognitiva de las emociones. Madrid: Siglo Veintiuno Editores (Trabajo original publicado en 1988).

Palacios, J. R., \& Bustos, J. M. (2013). Validez factorial de la autoeficacia ambiental y su influencia estructural sobre la conducta proambiental en jóvenes. Revista Iberoamericana de Diagnóstico y Evaluación - e Avaliação Psicológica, 35 (1), 95- 111.

Perugini, M., \& Bagozzi, R. P. (2004). An alternative view of pre-volitional processes in decision making: Conceptual issues and empirical evidence. En G. Haddock y G. R. Maio (Eds.). Contemporary Perspectives on the Psychology of Attitudes: The Cardiff Symposium (pp. 169-199). Hove, Reino Unido: Psychology Press.

Pinheiro, J., \& Corral-Verdugo, V. (2010). Time Perspective and sustainable Behavior. En V. Corral-Verdugo, C., García-Cadena, y M. Frías-Armenta (Eds.). Psychological Approaches to Sustainability: current trends in Theory, Research and Applications (pp. 205-224). Nueva York, EE.UU.: Nova Science Publishers.

Plutchik, R. (2001). The nature of emotions. Human emotions have deep evolutionary roots, a fact that may explain their complexity and provide tools for clinical practice. American Scientist, 89 (4), 344-350. 
Polak, E., \& McCullough, M. (2006). Is gratitude an alternative to materialism? Journal of Happiness Studies, 7, 343-360.

Reidl, L. (2005). Celos y envidia: Emociones humanas. Ciudad de México: Universidad Nacional Autónoma de México/Dirección General de Estudios de Posgrado/Programa de Maestría y Doctorado en Psicología / Facultad de Psicología.

Rodríguez, M., \& Padilla, V. (2008). Psicología de las emociones. En C. García, M. Muñiz y J. Montalvo. (Comps.), Conceptos de Psicología (pp. 133-173). México: Trillas.

Satorra, A., \& Bentler, P. M. (2001). A scaled Difference Chi-square test Statistic for moment structure analysis. Psychometrika, $66,507-514$.

Schmidt, C. (2008). Construcción de un cuestionario de emociones positivas en población entrerriana. Revista Iberoamericana de Diagnóstico y Evaluación - e Avaliação Psicológica, 26 (2), 117- 139.

Schmuck, P., \& Vlek, C. (2003). Psychologist can do much to support sustainable development. European Psychologist, 8, 66-76.

Scherer, K. R. (2004). Feelings integrate the central representation of appraisal-driven response organization in emotion. In A. S. R. Manstead, N. H. Frijda, y A. H. Fischer (Eds.), Feelings and emotions: The Amsterdam Symposium (pp. 136 -157). Cambridge, Inglaterra: Cambridge University Press.

Siemer, M., Mauss, I., \& Gross, J. J. (2007). Same situation-different emotions: How appraisals shape our emotions. Emotion, 7, 592-600.

Sevillano, V., Aragonés, J. I., \& Schultz, P. W. (2007). Perspective taking, environmental concern, and the moderating role of dispositional empathy. Environment $y$ Behavior, 39, 689-705.

Steg, L., \& Vlek, C. (2009). Social Science and Environmental Behaviour. En J. Boersema y L. Reijnders (Eds.), Principles of Environmental Sciences. (pp. 97-141). USA: Springer.

Syme, G., Porter, N., Goeft, U., y Kington, E. (2008). Integrating social well being into assessments of water policy: Meeting the challenge for decision makers. Water Policy, 10, 323-343.

Tapia-Fonllem, C., Corral-Verdugo, V., Gutiérrez-Sida, C., Mireles-Acosta, J., \& Tirado-Medina, H. (2010). Emotions and Proenvironmental Behavior. En V. CorralVerdugo, C. García-Cadena y M. FríasArmenta (Eds.), Psychological Approaches to Sustainability: current trends in theory, research and applications (pp. 249-267). New York, EE.UU.: Nova Science Publishers.

West, S. G., Finch, J. F., \& Curran, P. J. (1995). Structural equation models with nonnormal variables. En R. H. Hoyle (Ed.). Structural Equation Modeling. Concepts, Issues, and Applications (pp. 56-75). California, EE.UU.: Sage Publications.

White, M., Smith, A., Humphryes, K., Pahl, S., Snelling, D., \& Depledge, M. (2010). Blue space: The importance of water for preference, affect, and restorativeness ratings of natural and built scenes. Journal of Environmental Psychology, 30, 482-493. 